\title{
ALGUNAS BOLETACEAE QUE FRUCTIFICAN EN EL SUR DE CHILE
}

\author{
E. Valenzuela*, G. Moreno ** \& J. Grinbergs* \\ (*)Instituto de Microbiología. Facultad de Ciencias. Universidad \\ Austral de Chile Casilla 567. Valdivia. Chile. \\ (**)Departamento de Biología Vegetal (Botánica). Facultad de Ciencias \\ Universidad de Alcalá de Henares 28871 Madrid, España.
}

Palabras Clave: Boletaceae, Suillus tomentosus, Chile. Key Words: Boletaceae, Suillus tomentosus, Chile.

\section{RESUMEN}

Se citan cinco taxa de Boletaceae(s. lato) que fructifican en los bosques del Sur de Chile. Se aportan microfotografias de sus caracteres más importantesy se mencionan aspectos ecológicos. Suillus tomentosus (Kauffman) Singer Snell \& Dick, se cita por primera vez para Chile.

\section{INTRODUCCION}

Han sido citados para la micota chilena trece Boletaceae s. lato, de los cuales Boletus araucarianus Garrido, B. bresinskyanus Garrido, B. chilensis Sing., $\boldsymbol{B}$. loyita Horak, B. loyo Phil. ex Speg., B. putidus Horak y Gastroboletus valdivianus Horak, son especies autóctonas que fructifican en el bosque nativo de Araucaria araucana y Nothofagus (N. alpina, N. dombeyi, N. obliqua y $N$. procera), su sistemática, taxonomía y algunos aspectos ecológicos han sido estudiados por Philippi (1893), Espinoza $(1915,1916,1926)$, Singer $(1968,1969)$, Horak (1977). Mujica \& Oehrens (1980), Lazo (1983), (Garrido (1983, 1988) y Donoso (1990). Boletus edulis Bull.; Fr., Suillus granulatus (L.; Fr.) O. Kuntze, S. grevillei (Klotzch) Singer,S.luteus (L.: Fr.) S.F. Gray, Xerocomus chrysenteron (Bull.ex St. Amans) Quélet, Xerocumus cf. spadiceus (L.) Quelet y Phaeogyropus beniensis Singer \& Digilio, son taxa alóctonos que fructifican asociados a especies arbóreas (Betula pendula, Eucalyptus spp, Larixdecidua, Pinus pinaster, P. radiata, P. silvestris, P.spp, Psudotsuga menziesii, Salix spp. y Tsuga heterophylla ) que han sido introducidas en Chile, en su gran mayoría en forma ectomicorrícica y mayoritariamente con Pinus radiata, lo cual ha sido ampliamente estudiado y citado por numerosos au-

\section{SUMMARY}

[ Some Boletaceae that fructify in the southern forest of Chile]

Five Boletaceae (s. lato) species that fructify in the southern forests of Chile are recorded. Microphotographs of the most outstanding characters are added and some ecological aspects are mentioned. Suillus tomentosus (Kauffman) Singer, Snell \& Dick is reported for the first time for Chile.

tores entre ellos Singer \& Digilio $(1957,1960)$, Singer (1963,1964) Mikola (1969), Chu-Chou (1979), Peredo \& al. (1983), Lazo (1983), Garrido (1981, 1983, 1986, 1988), Garrido et al. (1982, 1983).

\section{MATERIAL Y METODOS}

Las preparaciones microscópicas han sido realizadas en hidróxido de sodio al $5 \%$, hidoxido de potasio al $10 \%$, rojo congo amoniacal, reactivo de Melzer y agua destilada. Se han fotografiado en un microscopio modelo Labophot (Nikon) con contraste de fases y sistema fotográfico automático incorporado. La película utilizada fué, kodak plus-X pan 125 ASA.

El material estudiado se conserva en el herbario del Departamento de Biología Vegetal (Botánica), de la Universidad de Alcalá de Henares (AH) y un duplicado en el herbario particular (E. Valenzuela) Universidad austral de Chile.

\section{RESULTADOS Y DISCUSION}

Boletus loyo Phil. ex Speg., An. Mus. Nac. Buenos Aires 23: 9 (1912). = Boletus loyus Espinosa, Bol. Mus. Nac. de Chile 8: 5 (1915). 
Material estudiado: En bosque nativo de Nothofagus. Chivèria, Valdivia. 17-IV-1991 A.H 14263.

Observaciones: Especie micorrizógena de Nothofagus (N. alpina, N.dombeyi, N.obliqua y N. procera) conocida solo de los alrededores de Concepción y Valdivia (Horak, 1977 y Garrido 1985). Por su parte Donoso (1990) señala que Boletus loyo ha sido recogido en renovales de Nothofagatus glauca en Bullileo.

Los carpóforos se caracterizan en la madurez por su gran robustez, el píleo puede llegar a medir hasta 35 $\mathrm{cm}$. de diámetro, de color rojo vinoso, tubos libres a emarginado-adnatos alrededor del estípite, este último es robusto de $8-15 \times 4-7 \mathrm{~cm}$., claviforme-ventrudo, de color amarillo volviéndose rojo-vinoso a púrpura y sin reticulaciones. Carne de color amarillo-crema.Sabor y olor a nueces frescas.

Es semejante a las especies Boletus regius Fr. o $B$. bicolor Peck.

Boletus loyo es una especie comestible, pero debido a la limitación geográfica (Horak, 1977), condiciones edáficas y climatológicas (Donoso, 1990) para el desarrollo de sus fructificaciones, solo se comercializa en pequeñas cantidades entre Valdivia, La Unión y otras zonas del sur de Chile. Respecto de las condiciones ecologicas, Donoso (1990) nos indica que cuando Nothofagus dombeyi se localiza fuera del bosque siempre verde particularmente hacia el E. y el N, B. loyo es reemplazado por otros mejores adaptados (hemos observado mayoritariamente especies de los géneros Amanita y Cortinarius) además B. loyo no es capaz de sobrevivir en áreas cubiertas con nieve durante el invierno (Singer \& Morello, 1960).

Suillus grevillei (Klotzch) Singer, Farlowia 2: 259(1945). =Boletus grevillei Klotzch, Linnaea 7: 198 (1832) . =Boletus elegans Fries, Epicr. Syst. Mycol.: 409-410 (1838).

Material estudiado: En suelo bajo Pinus sp. y Populus nigra cv Italica. Parque Saval, Valdivia 23 - XII - 1991. AH 1467. Bajo Larix decidua. Sierra Guadarrama, Leg.Soc. Micol. Castellana. Det. G. Moreno. AH 1363.

Macroscopía: Píleo de 5-15 cm. de diámetro, hemisférico, anchamente umbonado o convexo extendido. Cutícula viscosa, glabra de color amarillo-anaranjado o marrón-amarillo-anaranjado, el centro (disco) es de color marrón-ocráceo. Margen incurvado a planodecurvado en la madurez, concoloro al píleo. Tubos de $1-1.5 \mathrm{~cm}$. adnatos, subdecurrentes, de color amarillocitrino a amarillo-oliváceo. Poros débilmente angulosos, muy pequeños, 1-2 por mm., concoloros con los tubos y volviéndose marrones al roce o al corte. Estípite de
4-10 x 1-3 cm., sólido, cilíndrico a levemente claviforme hacia la base, de color amarillo-oro y con rugosidades de color marrón-anaranjado, hacia la base es de color verde azufre. Anillo apical, viscoso, cortiniforme, flocoso, de color blanco a blanco-amarillento, que delimita el estípite en dos porciones, la superior reticulada por la decurrencia de los tubos y la parte inferior de morfología fibrilosa. Carne al corte amarillenta, despuès rosado-grisácea y debilmente verdosa en la base del estípite. Sabor y olor un poco frutoso.

Microscopía: Esporađa ocrácea-olivácea-amarillenta.Esporas de 8-10 (11) x 3,5-4 (4,5) um, cilíndrico-elipsoidales, lisas, no amiloides, de color amarillento a ocráceo en $\mathrm{KOH} 10 \%$. Basidios de $25-32 \times 5-6,5$ um, claviformes, tetrásporicos, hialinos. Cistidios de (30) 40-60 × 6-7 (8) um, subcilíndricos a claviformes, algunos lageniformes, hialinos con material incrustante en la base, contenido ocráceo a amarillento en $\mathrm{KOH} 10 \%$. Caulocistidios solitarios o esparcidos en pequeños fascículos y con pigmento incrustante en $\mathrm{KOH}$ $10 \%$. Pileipellis filamentosa, formada por hifas cilíndricas, tabicadas de 3-6 um de diámetro rodeadas de una matríz gelatinosa, hialinas o con contenido amarillento-ocráceo pálido. Fíbulas ausentes.

Observaciones: Taxon descrito como ectomicorrízico con especies arbóreas del género Larix (Smith \& Thiers, 1971, Moreno \& al. 1986). Citada por vez primera para Chile por Garrido (1986) de Valdivia, asociada a Larix decidua.

En nuestros caso hemos recolectado los cuerpos fructíferos de esta especie entre Populus sp. y Pinus sp., estando más próximo a esta última especiearbórea. La asociación micorrizógena con especies del género Pinus está de acuerdo con la literatura consultada, así Garrido (1986) indica que ha sido demostrada la asociación con Pinus radiata.

Suillus grevellei de acuerdo a Moreno \& al. (1986), se considera un buen comestiole, teniendo la precaución de quitar la cutícula viscosa del píleo, lo cual debe hacerse en el campo.

Suillus luteus (L.: Fr.) Gray, Nat. Arrang. Brit. 1: 646 (1821).

=Boletus luteus Fries, Syst. Mycol. 1: 386 (1821).

Material estudiado: En bosque de Pinus radiata. Fundo Las Palmas, Valdivia 6-V-1990. AH 13975.

Bosque de Pinus sp. Parque SAVAL, Valdivia, 28-V. 1991. AH 14679.

Observaciones: Especie ectomicorrízica con Pinus 
pi naster, $P$. radiata, $P$. silvestris y Pseudotsuga menziesii. En nuestro país se encuentra ampliamente distribuida en plantaciones de las coníferas antes indicadas, donde ha sido estudiado ampliamente por Garrido (1981, $1983,1986,1988)$, Garrido \& al. $(1982,1983)$ y Peredo \& al. (1983).

Suillus tomentosus (Kauffman) Singer, Snell, \& Dick Mycologia 51: 570 (1960). (Figs. 1-7).

=Boletus tomentosus Kauffman, Pap. Mich. Acad. Sci. Arts \& Letters 1: 117 (1921).

$=$ Xerocomus lenticolor Snell \& Dick, Mycologia 52: 448 (1960).

Material estudiado: En humus de Pinus sp. y Abies sp. Jardín Botánico, Universidad Austral de Chile, Valdivia, 15-V-1990. AH 14677.

Macroscopía: Píleo de 4,4-10,2 cm. de diámetro, al principio convexo a anchamente convexo de color amarillo pálido o anaranjado-amarillo pálido de color más obscuro en la madurez, tomentoso flocoso, con escamas a escuámulas parduscas. Margen débilmente incurvado y con un tomento muy tosco. Poros pequeños de alrededor de 2 por $\mathrm{mm}$., de color marrón a vináceomarrón o amarillo-marrón. Tubos de $1-2 \mathrm{~cm}$. de longitud, adnatos a decurrentes de color amarillo pálido, en la madurez de color verde-amarillo sucio, se tiñen de color azul-verdoso al rozarlos o en los ejemplares envejecidos. Estípite de 3,3-10x1-2 cm, sólido, cilíndrico a claviforme, concoloro con el píleo o un poco más anaranjado, todo cubierto de gránulos, sin restos del velo universal. Carne de color amarillo sucio, volviéndose azul a azul-verdoso al corte. Sabor ligeramente ácido. Olor fúngico.

Microscopía: Esporas de 9,5-11,5 (12) x 3,5-4 um, fusiforme-elipsoidales, lisas, de color amarillo pálido. Basidios de 27-32 x 6-8 um, hialinos, claviformes y tetraspóricos. Pleurocistidios de 37-42 x 7-9 um, subcilíndricos a estrechamente claviformes de ápice obtuso, hialinos, de color amarillentos en $\mathrm{KOH} 10 \%$. Queilocistidios similares a los pleurocistidios frecuentemente con copioso pigmento amorfo de color marrón. Caulocistidios similares a los queilocistidios. PileipeHlis en las escuámulas formada por un trichodermis de hifas fusiformes y cilíndricas de 8-12 um de ancho, la mayoría con un contenido de color marrón. Fíbulas no observadas.

Observaciones: Píleo fibroso a escamoso, los poros cuando joven de color marrón, ausencia de velo, estípite con gránulos y la tendencia de los poros y la carne a teñirse de azul a azul-verdoso, al corte o al manipularlo forman la combinación de caracteres distintivos de esta especie. Se puede confundir con Suillus fuscotomentosus (Smith \& Thiers, 1971), pero en esta última especie la carne no gira al azul. Una especie europea muy próxima es Suillus variegatus el cual se diferencia por poseer el píleo afieltrado en la juventud, posteriormente cubierto de finas escuámulas (como arena espolvoreada), su estípite no es granuloso y su carne sólo azulea débilmente. Además fructifica con Pinus silvestris y $\boldsymbol{P}$. mugo en suelos ácidos y arenosos.

De acuerdo a Smith \& Thiers, (1971) y Arora (1986), esta especie se encuentra en forma dispersa a gregaria bajo Pinus spp. y otras coníferas, a finales del verano y principios del invierno, siendo muy común en la parte oeste de los Estados Unidos. Según Lincoff \& Knopf (1987) esta especie fructifica bajo Pinus de 2 y 3 acículas, entre Septiembre - Octubre en el Pacífico NW. y de Noviembre a Enero en California . Arora (1986) nos indica que fructifica con Pinus contorta y en bosques mixtos de Pinus y Populus, de acuerdo con este mismo autor la comestibilidad de esta seta es de mediocre calidad.

Xerocomuschrysenteron (Bull. ex St. Amans) Quélet, Fl. Mycol. 448 (1888). Fig. 8-10.

$=$ Boletus chrysenteron Bull. ex St. Amans, Fl. Agenaise 555 (1821).

Material estudiado: En suelo alrededor de tocones de Populus sp. Alameda de la Universidad Austral de Chile, Valdivia, 24-XI-1989. AH 13978.

Observaciones: De acuerdo a Moreno \& al. (1986), las características más constantes y fiables para diferenciar Xerocomus chrysenteron en el campo son la carne, subcuticular de color rojo-sangre, el estípite con gránulos muy pequeños de color rojizo carmín y la carne adquiere tonos azules según la humedad más o menos intensamente al rozamiento. Breitenbach \& Kränzlin (1991), indican que las formas precoces o tardías son difíciles de identificar.Xerocomus truncatus Sing., Snell \& Dick es una de las especies más próximas a $X$. chrysenteron que solo puede diferenciarse por poseer poro germinativo en elápice de la espora, mientras que carece de él; el $X$. chrysenteron, esta última es una especie cosmopolita, común, muy distribuída por todo el hemisferio boreal o septentrional y conocida también de América del Sur. Fructifica en bosques de coníferas y caducifolios en suelos ricos en humus $\mathrm{e}$ incluso sobre restos de madera descompuesta.

Especie citada para Chile, por Espinosa (1926), Singer (1969) y Garrido (1981), de acuerdo a Garrido 
(1986) este taxon se encuentra en nuestro país asociado en forma micorrízica con Betula pendula, Pinus radiata, Eucalyptus spp. y Salix spp. Garrido (1983) señala que Xerocomus chrysenteron recolectado en viveros forestales de Pinus radiata en la VIII Región del país prefiere para su desarrollo suelos arenosos, con una alta humedad (hasta $33 \%$ ) y pH 5.35-5.40, siendo dominante con Rhizopogon roseolus entre Abril y Mayo, luego de la declinación de Scleroderma citrinum. En nuestro caso hemos recolectado los cuerpos fructíferos de Xerocomus chrysenteron alrededor de tocones de Populus en la Alameda de la Universidad Austral de Chile, proliferando junto a Psathyrella candolleana y Coprinus disseminatus durante el mes de Noviembre, posteriormente (principios de Marzo de 1990) y alrededor de los mismos tocones de Populus, fructifica Paxillus involutus. Su distribución geográfica se localiza entre Concepción y Valdivia.

Gastronómicamente recogemos la cita de Moreno et al. (1986), quienes señalan que es una especie comestible, pero de calidad mediocre, porque su carne se pudre con facilidad. Algunos autores la definen de manera muy explícita como "un hongo comestible de carne muy blanda que carece de personalidad, perfume y sabor".

\section{Agradecimientos}

Queremos agradecer a la Agencia Española de Cooperacion Internacional del Ministerio de Asuntos Exteriores. Secretaría de Estado para la Cooperación Internacional y para Iberoamérica, la concesión a uno de nosotros (E. Valenzuela), de una beca para la realización del doctorado en la Universidad de Alcalá de Henares.

Agradecemos a L. García Gonzaga (Conservador del Herbario $\mathrm{AH}$ ). por su colaboración en el material solicitado

\section{REFERENCIAS}

Arora, D. , (1986). Mushrooms demystified a comprehensive guide to the fleshy fungi. Ed. Ten Speed Press. Berkeley.

Breitenbach,J., \& Kränzlin, E. (1991). Champignons de Suisse, contribution á la connaissance de la flore fongique de Suisse. Edition Mykologia Lucerne.

Chu-Chou,M., (1979). Mycorrhizal fungi of Pinus radiata in New Zealand. Soil Biol. Biochem. 11:557-562.

Donoso, C., (1990). Ecología Forestal.El bosquey su medio ambiente. Editorial Universitaria, Santiago de Chile.

Espinosa, M., (1915). Hongo chileno nuevo, el loyo Boletus loyus Espinosa, n. sp. Bol. Mus. Nac. Hist. Nat. 8: 5-11.

(1916). Contribución al conocimiento de los hongos chilenos. Bol. Mus.Nac. Hist. Nat. 9:65-94.

(1926). Enumeración de hongos chilenos. Rev. chilena Hist. Nat. 30: 297.

Garrido, N.,(1981) Contribución al conocimiento de los Agaricales (Mycota-Basidiomycetes) en plantaciones de Pinus radiata D. Don en la VIII Región de Chile. Tesis Dept. Botánica, Universidad de Concepcion, Chile.

(1983). Las Boletaceae en plantaciones de Pinus ra. diata D. Don en Chile (Fungi Basidiomycetes) Bol. Soc. Biol. Concepcion, Chile 54: 77-88.

(1985). Index Agaricalium chilcnsis. Biblioteca Mycologica 99. J. Cramer. Vaduz 339 pags.

(1986). Survey of ectomycorrhizal fungi associated with exotic forest tree in Chile. Nova Hedwigia 43:423-442.

Garrido, N. (1988). Agaricales s.l. und ihre mykorrhizen in den Nothofagus - wäldern mittelchiles. Biblioteca Mycologica 120.J. Cramer. 528 págs.

Garrido, N. \& Salas,J. (1982). Micorrizas ectotróficas en el establecimiento de plantaciones de Pinus radiata D. Don. In Schlatter, J. (ed). Evaluación de la productividad de sitios forestales. 22-24 Abril 1982. Universidad Austral de Chile, Chile. pp. 256-272.

Garrido,N., Marticorena,C., \& Oehrens, E. (1983). Ecological as pects and crop of Suillus luteus (Fungi, Boletaceae) in Pinus radiata forest in the Region of Bío-Bío, Chile. Bol. Soc. Biol. Concepcion 54: 89-101.
Horak,E,,(1977). New rare Boletus from Chile. Bol.Soc. Argen.Bot. 18: 97-109.

Lazo,W., (1983). Introducción al estudio de los hongos superiores II. Boletín Micológico 1: 77-119.

Lincoff, G. \& Knopf, A (1987). The audubon Society field guide to Nort American mushroorms. Ed. A. Chanticleer Press Edi tion.

Mikola, P., (1969). Mycorrhizal fungi of exotic forest plantations. Karstenia 10:169-175.

Moreno, G., Manjón, J. L. \& Zugaza, A, (1986). La guía de Incafo de los hongos de la Península Ibérica. Vols. Iy II. Incafo. Madrid.

Mujica, F. \& Oelrens, E., (1980). Flora fungosa chilena. $2^{\mathrm{a}}$.ed. Santiago.

Peredo, H., Oliva, M. \& Huber, A. (1983). Environmental factors determining the distribution in Pinus radiala grazing-forest plantations. Plant and Soil 71: 363-370.

Philippi, R.,(1963). Die pilze Chiles, soweit diselben als nahrungsmitel gebraucht werden. Hedwigia 32: 115 .

Singer, R., (1963). Ektroph, seine definition, geographische verber tung und bedeutung in der forstökologie mykorrhiza. Intern. Mykorthizas symposium. Weimar 1960. 223-231.

(1964). Boletes and related groups in South America. Nova Hedwigia 7: 69-71.

(1968). Nuevos hongos descubiertos en Chile. Biologicas. (Santiago) 49: 69-71. 29: $1-406$

(1969). Mycoflora Australis. Beih. Nova Hedwigia

Singer, R., \& Digilio,A.P.Ln,(1957). Las boletáceas austrosudamericanas. Lilloa 28:247-268.

(1960). Las boletáceas de Sudamérica tropical. Lilloa 30: 141.

Singer, R., \& Morello,J.H., (1960).Ectotrophic forest tree mycorrhizae and forest communities. Ecology 41: 549-551.

Smith, A.H., \& Thiers, H.D. (1971). The Boletes of Michigan. The University of Michigan press. 


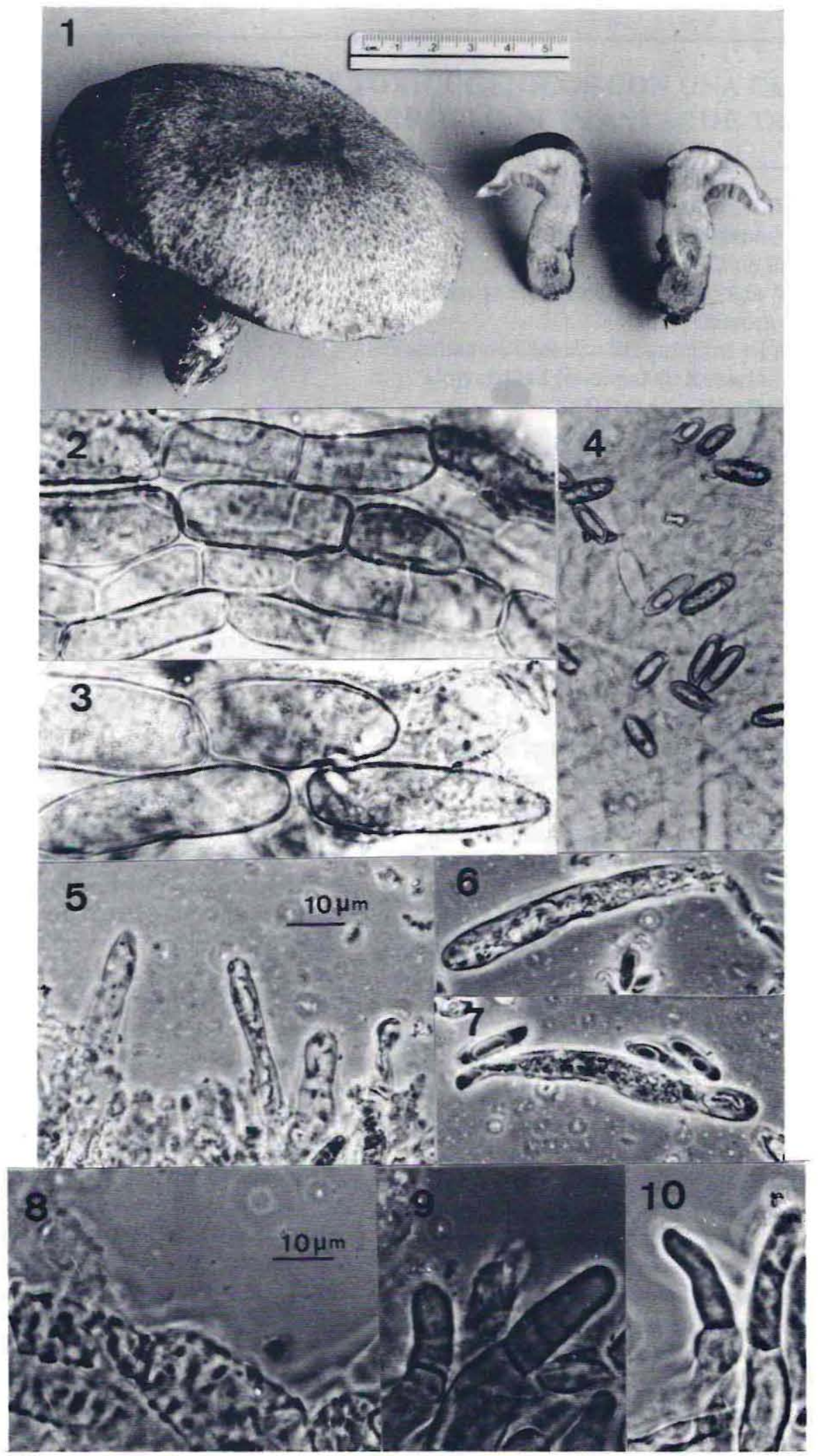

Figs. 1-7.-Suillus tomentosus (Kauffman) Singer, Snell \& Dick, AH 14677; 1: cuerpos fructiferos. 2 y 3: epicutis. 4: esporas. 5-7: cistidios himeniales.

Figs. 8-10.-Xerocomus chrysenteron (Bull. ex St. Amans) Quélet, AH 13978; 8: pigmento incrustante epicutis. 9-10: terminaciones epicutis. 